\title{
Efeitos da fragmentação florestal sobre vespas e abelhas solitárias em uma área da Amazônia Central
}

\author{
Elder F. Morato ${ }^{1}$ \\ Lúcio Antonio de O. Campos ${ }^{2}$
}

\begin{abstract}
Effects of forest fragmentation on solitary wasps and bees in an area in Central Amazonia. The effects of forest fragmentation on tree-hole nesting solitary wasps and bees were investigated at a site $90 \mathrm{~km}$ north of Manaus, Brazil. Wasp and bee faunas were monitored in continuous terra firme forest, forest fragments of 1,10 and $100 \mathrm{ha}$, natural gaps in continuous forest and deforested areas. These habitats were studied in terms of abundance, richness, diversity and similarity. The wasps and bees were monitored monthly during June 1988 through June 1990 by means of a trap-nests technique. A total of 1529 nests of wasps of 24 species and 405 nests of bees of 14 species were collected. The number of species of wasps and bees varied little among the habitats. The genus Trypoxylon Latreille, 1796 (Sphecidae) accounted for $79 \%$ of wasp nests and Centris Fabricius, 1804 (Anthophoridae) for $56 \%$ of bee nests. Wasps showed an overall preference for cleared areas and fragments of 1 ha, whereas bees showed an overall preference for continuous forest and natural gaps. Nevertheless, some species of wasps showed a preference for nesting in continuous forest and some bees a preference for deforested areas. Species found in deforested areas also nested in small size forest fragments. This shows that species occurring predominantly in cleared areas can also colonize small forest fragments. The diversity of wasps and bees was greater in continuous forest. The composition of wasp and bee faunas of continuous forest was different from that of altered habitats. The similarity between the natural gaps and cleared areas was the smallest. The data suggest that the forest adapted bees are more sensitive to habitat fragmentation than the corresponding species of wasps. It is concluded that the preexisting cavity nesting solitary wasp and bee communities were altered by the forest fragmentation. However, it appears that at least in part, the natural variation in continuous forest could be responsible for the results obtained from this study.

KEY WORDS. Amazonia, forest fragmentation, solitary bees, solitary wasps, trapnests
\end{abstract}

O desmatamento de florestas provoca, usualmente, a fragmentação da mata antes contínua, gerando o aparecimento de verdadeiras ilhas de matas isoladas umas das outras por áreas cobertas por pastagens ou por algum outro tipo de cultura. Além da perda de espécies provocada pela destruição da mata, podem ocorrer modificações nos fragmentos com o passar do tempo, em relação à diversidade e composição de sua fauna e flora, rompimento de antigas, e estabelecimento de novas interações entre as espécies, modificações nos processos biológicos e nas características do microclima e solo (LOVEJOY 1980).

1) Departamento de Ciências da Natureza, Universidade Federal do Acre. 69915-900 Rio Branco, Acre, Brasil.

2) Departamento de Biologia Geral, Universidade Federal de Viçosa. 36571-000 Viçosa, Minas Gerais, Brasil. 
O estudo das modificações na composição da fauna e flora dos fragmentos de mata após o isolamento é de grande importância para a adoção de critérios confiáveis para o estabelecimento de parques e reservas biológicas.

Vespas e abelhas são componentes muito importantes das comunidades, podendo ser muito sensíveis aos efeitos de perturbações ambientais. Alguns grupos de vespas solitárias são predadores de um grande número de insetos e aranhas (EVANS \& EBERHARD 1970). As abelhas, de um modo geral, são dependentes das plantas floríferas na obtenção de alimento e outros recursos. As plantas, por outro lado, dependem muitas vezes das abelhas para a sua reprodução, pois esses insetos são importantes promotores da polinização cruzada. Tanto vespas como abelhas são predados e parasitados por uma grande variedade de organismos (Evans \& EBERHARD 1970; BATRA 1984; ROUBIK 1989).

Vários gêneros de vespas das famílias Sphecidae, Eumenidae e Pompilidae e de abelhas das famílias Anthophoridae, Megachilidae e Apidae possuem espécies que fazem ninhos no interior de ramos ocos ou em cavidades preexistentes na madeira, muitas vezes feitas por besouros e outros insetos (KROMBEIN 1960a,b, 1967; Evans 1966; Evans \& EBERHARD 1970; CowAN 1991).

Tendo em vista a importância das vespas e abelhas como predadores e polinizadores e as relações que mantêm com outros organismos, este trabalho teve como objetivo estudar a comunidade desses insetos em fragmentos e áreas contínuas de floresta tropical, na Amazônia Central, procurando elucidar os efeitos da fragmentação da floresta sobre essas comunidades.

\section{MATERIAL E MÉTODOS}

\section{Descrição da área de estudo}

As coletas foram realizadas na Amazônia Central, em uma região situada aproximadamente a $70-90 \mathrm{~km}$ ao norte de Manaus, Amazonas $\left(2^{\circ} 30^{\prime} \mathrm{S}\right.$ e $\left.60^{\circ} \mathrm{W}\right)$, nas áreas de trabalho do "Projeto Dinâmica Biológica de Fragmentos Florestais" (PDBFF), convênio INPA/WWF/STRI, entre junho de 1988 e junho de 1990. Essa área encontra-se sob a jurisdição da Superintendência da Zona Franca de Manaus (SUFRAMA) e tem sido desmatada para a implantação de pastagens, visando o desenvolvimento da pecuária.

A região apresenta vegetação típica de floresta tropical úmida de terra firme, com dossel atingindo em média 30-37 m de altura. O sub-bosque é aberto e possui um grande número de palmeiras acaules do gênero Astrocaryum G. Mey. (HENDERSON et al. 1995). Os solos são em geral do tipo latossolos, pobres em nutrientes. O relevo é plano e ondulado, recortado por inúmeros igarapés. A precipitação média anual é de $2186 \mathrm{~mm}$, com uma estação mais seca entre julho e setembro, quando pode ser menor que $100 \mathrm{~mm}$ por mês (BIERREGAARD \& LOVEJOY 1988).

As coletas foram realizadas em dois fragmentos de mata nativa de 1 ha (códigos 1104 e 3114 ), dois fragmentos de 10 ha (1202 e 3209), um fragmento de 100 ha (3304) e em sete locais de mata contínua $(1113,1210,1301,1501 \mathrm{a}, 1501 \mathrm{~b}$, $1501 \mathrm{c}$ e MCPA). Ao longo deste texto, a expressão mata contínua será usada como sinônimo de floresta primária, não-fragmentada e não-perturbada. 
Os fragmentos de floresta primária foram isolados da mata contínua entre 1980 e 1983. A distância entre eles e a área de mata contínua mais próxima varia entre 100 a $650 \mathrm{~m}$.

Além dessas áreas, também foram feitas amostragens em duas áreas onde a vegetação nativa foi derrubada, situadas próximas dos fragmentos isolados. Essas áreas de derrubada serão, denominadas DERCO (Derrubada situada na Fazenda Esteio, próxima do acampamento Colosso) e DERPA (Derrubada situada na Fazenda Porto Alegre).

A derrubada DERCo é constituída de pastagem e de vegetação secundária com várias espécies de plantas ruderais. A DERPA é constituída de vegetação secundária (capoeira) muito desenvolvida e fechada, dominada por espécies de Cecropia Loefl. (Cecropiaceae). Assim, três fragmentos estudados 3114 (1 ha), 3209 (10 ha) e 3304 (100 ha) estavam separados da mata contínua por uma capoeira alta e espessa, dominada por espécies de Cecropia. Já os dois outros fragmentos 1104 (1 ha) e 1202 (10 ha) estavam separados da mata contínua por vegetação baixa e pasto.

Uma das áreas de mata contínua estudada (1501) possuía um amplo sistema de trilhas, o que facilitava o deslocamento e a localização dos pontos amostrais. Nesta área foram também realizadas coletas em 23 clareiras naturais, com áreas entre 25 e $40 \mathrm{~m}^{2}$ aproximadamente, produzidas pela queda de árvores. $\mathrm{O}$ interior dessas clareiras era mais iluminado que o sub-bosque adjacente e possuía troncos em decomposição e arbustos de pequeno porte. Nas outras áreas de mata contínua estudadas não foram amostradas clareiras.

\section{Amostragem por ninhos-armadilhas}

A amostragem de vespas e abelhas solitárias foi realizada com o emprego de ninhos-armadilhas. Duas peças de madeira de $25 \times 35 \times 120 \mathrm{~mm}$ foram unidas em uma morça e furadas longitudinalmente de forma que, quando as duas metades da peça estão unidas, forma-se um orifício com diâmetro de 4,$8 ; 9,5$ ou $12,7 \mathrm{~mm}$ e 8 $\mathrm{cm}$ de comprimento. No campo, as duas metades foram unidas por fita adesiva. Foi utilizada madeira de Pithecellobium Martius (Leguminosae) por ser inodora e não soltar fibras com facilidade.

No interior de cada fragmento isolado ou local de mata contínua, foram escolhidas quatro árvores separadas entre si por uma distância máxima de $50 \mathrm{~m}$. Cada conjunto de quatro árvores constituiu uma unidade amostral. Em cada árvore foram colocados três blocos de ninhos-armadilhas; um bloco a $1,5 \mathrm{~m}$; um bloco a 8 $\mathrm{m}$ e outro a $15 \mathrm{~m}$ de altura. Cada bloco continha nove ninhos-armadilhas, três de cada diâmetro, distribuídos ao acaso. Os blocos foram atados a um fio de náilon que passava por uma roldana de metal presa à árvore, a $15 \mathrm{~m}$ de altura. Isso possibilitou a descida, verificação e suspensão dos blocos periodicamente.

Nas derrubadas (DERCO e DERPA) foram colocados 20 blocos de ninhos-armadilhas a $1,5 \mathrm{~m}$ de altura. Nas clareiras foram colocados 23 blocos, um em cada clareira, também a $1,5 \mathrm{~m}$ de altura. 
Os ninhos-armadilhas foram inspecionados, quinzenalmente, entre junho de 1988 e junho de 1990; aqueles ocupados por ninhos de vespas ou abelhas foram retirados e substituídos por peças vazias. As peças ocupadas foram levadas para o laboratório, onde os ninhos foram abertos, separadas suas duas metades e, em seguida, descritos. Após a descrição, os ninhos foram colocados em local apropriado até a emergência dos insetos adultos; a seguir, foram limpos e reutilizados no campo. Assim, ninhos, dos quais emergiram adultos, foram identificados com relação às vespas ou abelhas que os construíram e provisionaram. Ninhos, dos quais não ocorreram emergências, foram identificados, com base em suas características, por comparação com ninhos semelhantes.

\section{Análise dos dados}

Como o esforço de coleta (número de ninhos-armadilhas instalados) foi diferente em alguns ambientes, grande parte dos resultados apresentados em forma gráfica neste trabalho e também dos dados utilizados em algumas análises foram corrigidos. Essas correções foram realizadas com a finalidade de padronizar o esforço de coleta nos diferentes ambientes e, consequentemente, evitar distorções de amostragem. Tomou-se como padrão o esforço de coleta realizado nos fragmentos de 1 ha, 10 ha e clareiras naturais ( 216 ninhos-armadilhas foram instalados nestes ambientes).

Em algumas análises, não foram incluídos os dados coletados no fragmento de mata de 100 ha, em razão de problemas de amostragem, uma vez que não havia réplicas de fragmentos com este tamanho.

Valores de diversidade, em espécies de vespas e abelhas, foram calculados para os ambientes estudados, pelo índice de Shannon (MAGURRAN 1988).

Os valores obtidos por esse índice foram comparados por uma variação do teste t, proposta por HUTCHESON (1970). Esta comparação envolve, inicialmente, o cálculo das variâncias dos índices de Shannon obtidos a partir das amostras a serem comparadas. As variâncias e os valores dos índices das amostras são, então, utilizados numa fórmula que permite o cálculo de "t". Os graus de liberdade são obtidos a partir das variâncias e do número de ninhos das amostras que estão sendo comparadas.

Valores de equitabilidade em espécies de vespas e abelhas foram calculados, para os ambientes estudados, pelo índice J' de Pielou (LUDWIG \& REYNOLDS 1988).

A similaridade faunística entre os ambientes estudados, em relação às espécies de vespas e abelhas coletadas, foi verificada pelo coeficiente de dissimilaridade "distância corda" (PIELOU 1984; LUDWIG \& REYNOLDS 1988), o qual possui um intervalo de variação compreendido entre 0,00 (maior similaridade) e 1,44 (menor similaridade).

Com os valores obtidos, foram montados dendrogramas de dissimilaridade, de acordo com o método de agrupamento UPGMA (LUDWIG \& REYNOLDS 1988).

Foi calculada a correlação entre os valores de dissimilaridade, obtidos entre os ambientes estudados, em relação à fauna de vespas e em relação à fauna de abelhas pelo coeficiente de Pearson (r) (SOKAL \& ROHLF 1981). A significância do valor do coeficiente foi testada pelo teste " $t$ ". 


\section{RESULTADOS}

\section{Riqueza e abundância das espécies}

Foram coletados 1529 ninhos de 24 espécies de vespas pertencentes às famílias Sphecidae, Eumenidae e Pompilidae. A relação das espécies coletadas, bem como os resultados para cada unidade amostral, são apresentados de forma detalhada na tabela I.

A família Sphecidae foi a mais representada nas coletas, 1507 ninhos de 17 espécies. $\mathrm{O}$ gênero Trypoxylon Latreille, 1796 foi o mais abundante, 1191 ninhos de 11 espécies (79\%), sendo o subgênero Trypargilum Richards, 1934 o mais comum, com 1178 ninhos de oito espécies $(78,2 \%)$.

As espécies mais abundantes foram Trypoxylon (Trypargilum) lactitarse Saussure, 1867 (33\%) e T. (Trypargilum) nitidum F. Smith, 1856 (30\%).

Espécies de vespas, como um todo, fundaram mais ninhos em locais de derrubada e fragmentos de 1 ha (Tab. II). O número de ninhos fundados variou muito entre os locais de coleta, sendo maior na derrubada DERCO e menor na mata contínua 1210 (Tab. I).

O número de espécies coletadas nos diferentes ambientes foi pequeno (Tab. II). Foram coletadas mais espécies de vespas na mata contínua (16) do que nos fragmentos de mata e derrubada.

O número de ninhos fundados por abelhas foi menor que o número fundado por vespas. Foram coletados 405 ninhos de 14 espécies (Tab. I). As abelhas coletadas pertencem às famílias Anthophoridae, Megachilidae e Apidae. A família Anthophoridae foi a mais comum, com 232 ninhos fundados, distribuídos entre cinco espécies. Destes, 98,3\% foram fundados por espécies do gênero Centris Fabricius, 1804.

As espécies mais abundantes foram Centris terminata Smith, 1874 (32\%), C. dichrootricha Moure, 1945 (19,8\%), Megachile orbiculata Mitchel, $1929(18 \%)$ e Anthodioctes moratoi Urban, 1999 (15,1\%).

Constatou-se a tendência, embora pouco acentuada, para uma maior fundação de ninhos de abelhas em locais de mata contínua e no interior de clareiras naturais (Tab. II). Maior número de ninhos foi coletado nas clareiras (78) e menor na derrubada DERPA (6). Mais espécies foram coletadas na mata contínua que nos demais ambientes.

Algumas espécies de vespas foram freqüentes em todos os locais de coleta. Outras fundaram ninhos com maior freqüência em apenas alguns locais. Trypoxylon (Trypargilum) nitidum fundou ninhos em todos os locais de coleta. Houve maior abundância dessa espécie nos fragmentos de 1 e 10 ha e nos locais de derrubada. Trypoxylon (Trypargilum) lactitarse fundou ninhos principalmente em locais de derrubada $(60,4 \%)$. O número de ninhos fundados na derrubada DERCO foi muito maior que na derrubada DERPA (Tab. I).

As espécies de abelhas Megachile orbiculata, A. moratoi e Centris terminata fundaram ninhos, principalmente, na mata contínua e nas clareiras naturais. Já $C$. dichrootricha fundou ninhos, principalmente, na derrubada e nos fragmentos de 1 e 10 ha. 
Tabela I. Número de ninhos de espécies de vespas e abelhas solitárias coletados em locais de mata continua, fragmentos de mata, áreas desmatadas (pastagem e capoeira) e clareiras naturais entre junho de 1988 e junho de 1990 nas áreas do PDBFF, Manaus, Amazonas. (*) Os números entre parênteses referem-se ao total de ninhos-armadilhas instalados em cada local de amostragem; $\left({ }^{* *}\right)$ DERCO: área amostrada de derrubada ocupada por pastagem na Fazenda Esteio; DERPA: área amostrada de derrubada ocupada por capoeira na Fazenda Porto Alegre; MCPA: área de mata contínua amostrada na Fazenda Porto Alegre; 1501a, 1501b e 1501c: áreas de mata continua amostradas na reserva 1501.

\begin{tabular}{|c|c|c|c|c|c|c|c|c|c|c|c|c|c|c|c|c|}
\hline \multirow[t]{2}{*}{ Espécie } & \multicolumn{2}{|c|}{$\begin{array}{l}\text { Derrubada } \\
\quad(180)\end{array}$} & \multicolumn{2}{|c|}{$\begin{array}{l}\text { Frag. 1ha } \\
(216)\end{array}$} & \multicolumn{2}{|c|}{$\begin{array}{l}\text { Frag. 10ha } \\
\quad(216)\end{array}$} & \multirow{2}{*}{$\begin{array}{l}\begin{array}{c}\text { Frag. 100ha } \\
(108)\end{array} \\
3304\end{array}$} & \multicolumn{7}{|c|}{$\begin{array}{l}\text { Mata continua } \\
\quad(756)\end{array}$} & \multirow[t]{2}{*}{$\begin{array}{c}\text { Clareiras naturais } \\
(216)\end{array}$} & \multirow[t]{2}{*}{ Total } \\
\hline & DERCO & DERPA & 1104 & 3114 & 1202 & 3209 & & 1113 & 1210 & 1301 & $1501 \mathrm{a}$ & $501 \mathrm{~b}$ & $1501 \mathrm{c}$ & ICPA & & \\
\hline \multicolumn{17}{|l|}{ Sphecidae } \\
\hline Ampulex elegantula Kohl, 1893 & & 2 & & & & & & & & & & & 1 & & & 3 \\
\hline Pison (Entomopison) sp. & & & & & & & & & & & 1 & & & & & 1 \\
\hline P. (Pison) cressoni Rohwer, 1911 & 8 & & & & & & & & & & & & & & & 8 \\
\hline Pisoxylon xanthosoma Menke, 1968 & & & & & & & 1 & 2 & & & 4 & 5 & 1 & & 4 & 17 \\
\hline Podium rufipes Fabricius, 1804 & & & 1 & 11 & 10 & 18 & 34 & 6 & 13 & 14 & 15 & 5 & 24 & 17 & 17 & 185 \\
\hline P. sexdentatum Taschenberg, 1869 & & & & 1 & 7 & 2 & & 15 & 2 & 10 & 21 & 2 & 22 & & 4 & 86 \\
\hline Trypoxylon (Trypargilum) nitidum F. Smith, 1856 & 35 & 29 & 79 & 44 & 38 & 25 & 23 & 24 & 9 & 44 & 25 & 21 & 21 & 27 & 15 & 459 \\
\hline T. (Trypargilum) lactitarse Saussure, 1867 & 222 & 83 & 27 & 103 & 26 & 14 & & 1 & 1 & 7 & & 1 & 1 & 12 & 7 & 505 \\
\hline T. (Trypargilum) fugax Fabricius, 1804 & 4 & & & & & & & & & & & & & & & 4 \\
\hline T. (Trypargilum) xanthandrum Richards, 1934 & & & & & 1 & & & & & & & & & & & 1 \\
\hline T. (Trypargilum) aff. nitidum & 1 & 1 & 2 & 4 & 3 & 1 & 2 & & 3 & 9 & 4 & & & 16 & & 46 \\
\hline T. (Trypargilum) sp.1 & & & & & & 2 & & & & & 4 & & & 2 & & 8 \\
\hline T. (Trypargilum) sp.2 & & 14 & 18 & 1 & 7 & 19 & & 1 & 1 & 2 & 30 & 23 & 11 & 8 & 35 & 170 \\
\hline T. (Trypargilum) scrobiferum Richards, 1934 & & & & & & & & & & & 2 & & & & & 2 \\
\hline T. (Trypoxylon) punctivertex Richards, 1934 & & 1 & 4 & 1 & & & & & & & & & & & & 6 \\
\hline T. (Trypoxylon) aff. ungicome & & & & & & & & & & 1 & & & & & 4 & 5 \\
\hline T. (Trypoxylon) sp. & & & & & & & 1 & & & & & & & & & 1 \\
\hline \multicolumn{17}{|l|}{ Pompilidae } \\
\hline Auplopus sp. & & & & & 1 & 1 & & & & & 1 & & & 1 & 1 & 5 \\
\hline Priochilus sp. & & 1 & & & & & & & 2 & & & 2 & & & & 5 \\
\hline \multicolumn{17}{|l|}{ Eumenidae } \\
\hline Ancistroceroides sp. & & & & & & 1 & & & & & & & & & & 1 \\
\hline Pachodynerus gianelli (Gribodo, 1891) & 4 & & & & & & & & & & & & & & & 4 \\
\hline Pachodynerus sp.1 & & & & & & & & 1 & & & 1 & & & & 2 & 4 \\
\hline Pachodynerus sp.2 & & & & & 2 & & & & & & & & & & & 2 \\
\hline Zethus sp. & & & & & & & & & & 1 & & & & & & 1 \\
\hline Número de ninhos & 274 & 131 & 131 & 165 & 95 & 83 & 61 & 50 & 31 & 88 & 108 & 59 & 81 & 83 & 89 & 1529 \\
\hline Número de espécies & 6 & 7 & 6 & 7 & 9 & 9 & 5 & 7 & 7 & 8 & 11 & 7 & 7 & 7 & 9 & 24 \\
\hline
\end{tabular}

Continua 
Tabela I. Continuação.

\begin{tabular}{|c|c|c|c|c|c|c|c|c|c|c|c|c|c|c|c|c|}
\hline \multirow[t]{2}{*}{ Espécie } & \multicolumn{2}{|c|}{$\begin{array}{l}\text { Derrubada } \\
\quad(180)\end{array}$} & \multicolumn{2}{|c|}{$\begin{array}{l}\text { Frag. 1ha } \\
\text { (216) }\end{array}$} & \multicolumn{2}{|c|}{$\begin{array}{l}\text { Frag. 10ha } \\
\quad(216)\end{array}$} & \multirow{2}{*}{$\begin{array}{c}\begin{array}{c}\text { Frag. 100ha } \\
(108)\end{array} \\
3304\end{array}$} & \multicolumn{7}{|c|}{$\begin{array}{l}\text { Mata continua } \\
\quad(756)\end{array}$} & \multirow[t]{2}{*}{$\begin{array}{c}\text { Clareiras naturais } \\
(216)\end{array}$} & \multirow[t]{2}{*}{ Tota } \\
\hline & DERCO & DERPA & 1104 & 3114 & 1202 & 3209 & & 1113 & 1210 & 1301 & $1501 a$ & $1501 b$ & 15010 & MCPA & & \\
\hline \multicolumn{17}{|l|}{ Apidae } \\
\hline Eufriesea purpurata (Mocsáry, 1896) & & & & & & & & & & & & & & & 1 & 1 \\
\hline Euglossa gaianii Dressler, 1982 & 1 & 1 & & 1 & & & & 2 & 1 & & 3 & & 2 & 1 & & 12 \\
\hline Euplusia theresiae (Mocsáry, 1908) & & & & & & & & & & & & & & & 2 & 2 \\
\hline \multicolumn{17}{|l|}{ Anthophoridae } \\
\hline Centris dichrootricha Moure, 1945 & 11 & 4 & 11 & 17 & 6 & 7 & 1 & 3 & 2 & 3 & 1 & 2 & 2 & 6 & 4 & 80 \\
\hline C. analis (Fabricius, 1804) & 9 & & 1 & 1 & & & & 1 & & 1 & & 1 & 2 & & & 16 \\
\hline C. terminata Smith, $1874^{\circ}$ & 2 & 1 & & 9 & 2 & 8 & 6 & & 9 & 9 & 5 & 16 & 7 & 21 & 34 & 129 \\
\hline C. bicornuta Mocsáry, 1899 & & & & & & 3 & & & & & & & & & & 3 \\
\hline Lagobata ornata Spinola, 1851 & & & & & & & & 1 & 3 & & & & & & & 4 \\
\hline \multicolumn{17}{|l|}{ Megachilidae } \\
\hline Anthodioctes moratoi Urban, 1999 & & & 4 & & 1 & 4 & 1 & 17 & 4 & 3 & 5 & 4 & 6 & 7 & 5 & 61 \\
\hline Duckanthidium sp.1 & & & & 1 & & & & & & & & & & & & 1 \\
\hline Duckanthidium sp.2 & & & & 2 & 2 & & & 2 & & & & & & & 1 & 7 \\
\hline Megachile orbiculata Mitchel, 1929 & & & & & & & 7 & & 10 & 5 & 7 & 4 & 7 & 2 & 31 & 73 \\
\hline M. (Rhysochile) cfr. cara & & & & & & & & 1 & & & & & & & & 1 \\
\hline Megachile sp. & & & 2 & & 1 & & 3 & & & & & & & & & 6 \\
\hline Número de ninhos & 23 & 6 & 18 & 31 & 12 & 22 & 18 & 27 & 30 & 23 & 25 & 27 & 26 & 39 & 78 & 405 \\
\hline Numero de espécies & 4 & 3 & 4 & 6 & 5 & 4 & 5 & 7 & 6 & 5 & 5 & 5 & 6 & 5 & 7 & 14 \\
\hline Número total de ninhos & 297 & 137 & 149 & 196 & 107 & 105 & 79 & 77 & 61 & 111 & 133 & 86 & 107 & 122 & 167 & 1934 \\
\hline Número total de espécies & 10 & 10 & 10 & 13 & 14 & 13 & 10 & 14 & 13 & 13 & 16 & 12 & 13 & 12 & 16 & 38 \\
\hline
\end{tabular}


Tabela II. Abundância de vespas e abelhas solitárias ( $n^{\circ}$ de ninhos) (A) e indices de riqueza $(S)$, diversidade, $\left(H^{\prime}\right)$ e equitabilidade $\left(J^{\prime}\right)$ dos ambientes estudados.

\begin{tabular}{|c|c|c|c|c|c|c|c|c|}
\hline \multirow{2}{*}{ Ambientes } & \multicolumn{4}{|c|}{ Vespas solitárias } & \multicolumn{4}{|c|}{ Abelhas solitárias } \\
\hline & A & $\mathrm{s}$ & $H^{\prime}$ & J' & A & $\mathrm{s}$ & $H^{\prime}$ & $J^{\prime}$ \\
\hline Mata Continua & 500 & 16 & 1,861 & 0,671 & 197 & 10 & 1,767 & 0,767 \\
\hline Clareiras & 89 & 9 & 1,779 & 0,773 & 78 & 7 & 1,263 & 0,649 \\
\hline Frag. 10 ha & 178 & 11 & 1,721 & 0,718 & 34 & 6 & 1,494 & 0,834 \\
\hline Frag. 1 ha & 296 & 7 & 1,199 & 0,617 & 49 & 9 & 1,414 & 0,644 \\
\hline Derrubada & 405 & 10 & 0,872 & 0,379 & 29 & 4 & 1,123 & 0,810 \\
\hline
\end{tabular}

Houve as seguintes espécies de vespas que nidificaram apenas ou em maior freqüência na mata contínua (incluindo as clareiras): Podium sexdentatum Taschenberg, 1869, P. rufipes Fabricius, 1804, T. (Trypargilum) aff. nitidum, T. (Trypargilum) scrobiferum Richards, 1934, T. (Trypargilum) sp.2, T. (Trypoxylon) aff. ungicorne, Pisoxylon xanthosoma Menke, 1968, Pison (Entomopison) sp., Pachodynerus sp., Zethus sp. e Auplopus sp. As abelhas que também fundaram ninhos em maior freqüência ou apenas na mata contínua foram as seguintes: $C$. terminata, Lagobata ornata Spinola, 1851, A. moratoi, Duckanthidium sp., M. orbiculata, Megachile (Rysochile) cfr.cara, Eufriesea purpurata (Mocsáry, 1896) e E. theresiae (Mocsáry, 1908). O número de ninhos fundados por estas espécies, corrigido pelo esforço de coleta, nos diferentes ambientes é mostrado na figura 1 .

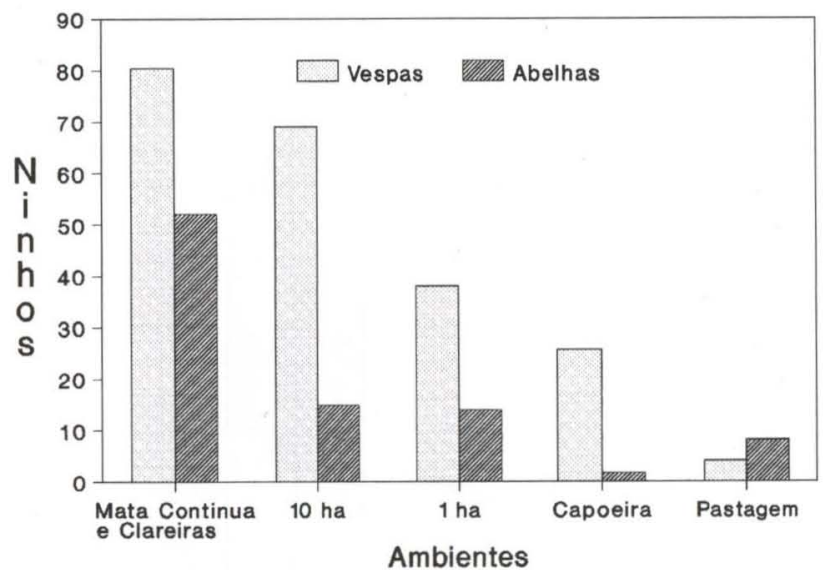

Fig. 1. Número de ninhos coletados de espécies de vespas e abelhas mais comuns na mata contínua, corrigido pelo esforço de coleta, em diferentes ambientes.

A fundação de ninhos por estas vespas e abelhas foi bastante reduzida nos fragmentos de 1 ha, em relação à mata contínua (redução de $52,7 \%$ e $73,1 \%$, respectivamente). Em fragmentos de 10 ha essa redução foi muito menor para vespas do que para abelhas $(14,1 \%$ e $71,2 \%$, respectivamente). A fundação de ninhos dessas vespas na pastagem foi significativamente menor que na capoeira $\left(X^{2}=16,1 ; p<\right.$ $0,01)$. 
Contudo, as seguintes espécies de vespas foram muito mais comuns na derrubada e nos fragmentos de mata do que na mata contínua (incluindo as clareiras): Ampulex elegantula Kohl, 1893, Pison cressoni Rohwer, 1911, T. (Trypargilum) nitidum, T. (Trypargilum) lactitarse, T. (Trypargilum) fugax Fabricius, 1804, T. (Trypoxylon) punctivertex Richards, 1934 e algumas espécies de Eumenidae. As abelhas $C$. dichrootricha e $C$. analis (Fabricius, 1804) foram também muito mais freqüentes na derrubada e fragmentos de mata do que na mata contínua. $\mathrm{O}$ número de ninhos fundados por estas espécies, corrigido pelo esforço de coleta, nos diferentes ambientes é mostrado na figura 2 .

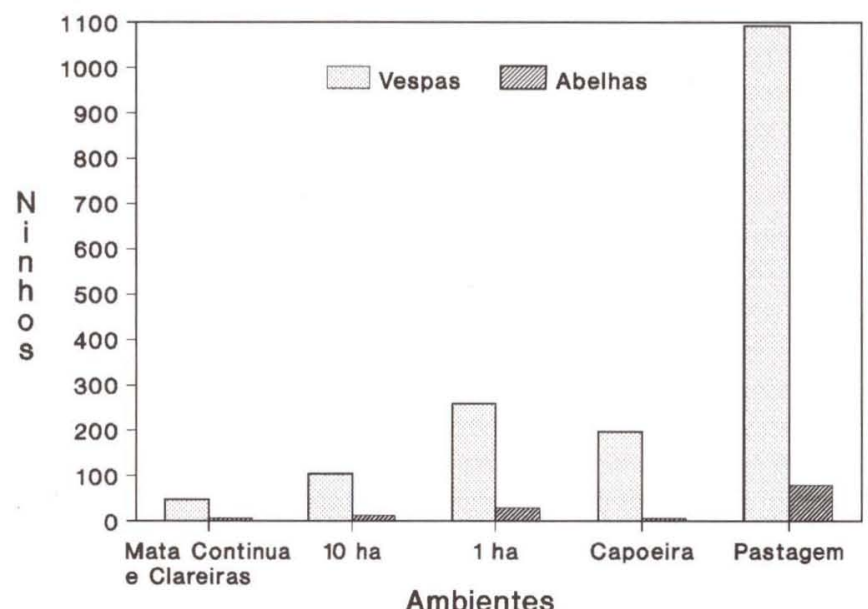

Fig. 2. Número de ninhos coletados de especies de vespas e abelhas mais comuns na área de pastagem, corrigido pelo esforço de coleta, em diferentes ambientes.

Ao contrário das vespas e abelhas mais típicas da mata contínua, a fundação de ninhos por esse grupo foi mais freqüente nas áreas de pastagem, capoeira e fragmentos de 1 ha. Houve uma acentuada diminuição nos fragmentos de 10 ha e mata contínua. $\mathrm{O}$ número de ninhos dessas vespas na pastagem foi significativamente maior do que na capoeira $\left(X^{2}=621,4 ; p<0,001\right)$. O mesmo ocorreu para as abelhas $\left(X^{2}=61,5 ; p<0,001\right)$. A redução na fundação de ninhos de vespas da pastagem para os fragmentos de 1 e 10 ha foi de $76,4 \%$ e $90,6 \%$, respectivamente. Para as abelhas esses valores foram de $62,5 \%$ e $83,8 \%$, respectivamente.

\section{Diversidade de espécies nos ambientes}

Os valores de diversidade e equitabilidade de espécies de vespas e abelhas nos ambientes estudados são mostrados na tabela II.

Considerando a diversidade de espécies de vespas, não houve diferença significativa entre a mata continua e as clareiras naturais $(t=0,802 ; \mathrm{p}>0,10)$. Contudo, a diversidade da mata contínua foi significativamente maior que a dos fragmentos de 10 ha, 1 ha e derrubada $(t=1,827 ; 10,015$ e 13,680, respectivamente; $p<0,05)$. A diversidade das clareiras não foi significativamente diferente da do fragmento de 10 ha $(t=0,511 ; p>0,10)$, mas foi maior que a do fragmento de 1 ha 
e a da derrubada $(p<0,001)$. A diversidade do fragmento de 10 ha foi significativamente maior que a do fragmento de 1 ha $(\mathrm{t}=6,300 ; \mathrm{p}<0,001)$ e da derrubada ( $\mathrm{t}$ $=9,7 ; \mathrm{p}<0,001)$. A do fragmento de 1 ha foi significativamente maior que a da derrubada $(\mathrm{t}=4,190 ; \mathrm{p}<0,001)$.

Em relação à diversidade de abelhas, a mata contínua apresentou uma diversidade maior que as clareiras, os fragmentos de 10 ha, 1 ha e a derrubada ( $p<$ $0,05)$. Com relação aos fragmentos de 10 ha, 1 ha, clareiras e derrubada, a única diferença significativa ocorreu entre o fragmento de 10 ha e a derrubada $(t=2,310$; $\mathrm{p}<0,05)$.

\section{Similaridade entre os ambientes}

Na tabela III são apresentados os valores de dissimilaridade entre os ambientes estudados, em relação à fauna de vespas e abelhas. Em relação à fauna de vespas, os valores mais altos de similaridade ocorreram entre os fragmentos de 1 ha e 10 ha, entre o de 10 ha e a mata contínua, entre o de 1 ha e a derrubada, e entre a mata contínua e as clareiras naturais. A similaridade entre a mata contínua, os fragmentos de 1 ha e a derrubada foi pequena, o mesmo ocorrendo com as clareiras naturais. A maior dissimilaridade ocorreu entre a derrubada e as clareiras naturais do interior da floresta. $\mathrm{O}$ dendrograma de dissimilaridade entre os ambientes é mostrado na figura 3 . A derrubada e as clareiras naturais apresentaram pequena similaridade com o grupo formado pelos fragmentos de 1 e 10 ha e a mata contínua.

Tabela III. Dissimilaridade, em relação a espécies de vespas e abelhas solitárias, entre os ambientes, calculada pelo índice de "distância corda"*. As comparaçōes foram organizadas pela similaridade em ordem decrescente.

\begin{tabular}{lc}
\hline \multicolumn{1}{c}{ Ambientes } & Coeficiente de dissimilaridade \\
\hline Em espécies de vespas & \\
Frag. 1 ha $X$ frag. 10 ha & 0,44 \\
Frag. 10 ha $X$ mata continua & 0,45 \\
Frag. 1 ha $X$ derrubada & 0,55 \\
Mata continua $X$ clareiras & 0,70 \\
Frag. 10 ha $X$ clareiras & 0,73 \\
Frag. 1 ha $X$ mata continua & 0,82 \\
Frag. 10 ha $X$ derrubada & 0,86 \\
Frag. 1 ha $X$ clareiras & 1,03 \\
Mata continua $X$ derrubada & 1,21 \\
Clareiras $X$ derrubada & 1,21 \\
Em espécies de abelhas & \\
Frag. 1 ha $X$ frag. 10 ha & 0,42 \\
Frag. 1 ha $X$ derrubada & 0,50 \\
Mata continua $X$ clareiras & 0,53 \\
Frag. 10 ha $X$ mata continua & 0,75 \\
Frag. 10 ha $X$ derrubada & 0,75 \\
Frag. 10 ha $X$ clareiras & 0,99 \\
Frag. 1 ha $X$ mata continua & 1,01 \\
Mata continua $X$ derrubada & 1,15 \\
Frag. 1 ha $X$ clareiras & 1,17 \\
Derrubada $X$ clareiras & 1,27 \\
\hline
\end{tabular}

$\left.{ }^{\star}{ }^{\star}\right)$ Este índice varia entre 0 e 1,42.

Revta bras. Zool. 17 (2): 429 - 444, 2000 


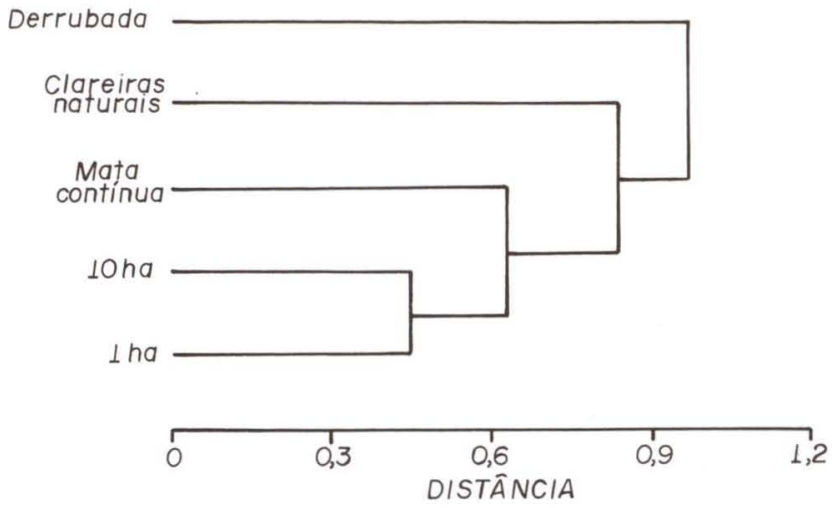

Fig. 3. Dendrograma de dissimilaridade entre os ambientes, em relação à fauna de vespas.

Em relação à fauna de abelhas, os maiores valores de similaridade ocorreram entre os fragmentos de 1 ha e 10 ha, entre o de 1 ha e a derrubada e entre a mata contínua e as clareiras naturais. Maior dissimilaridade ocorreu entre o fragmento de 1 ha e a mata contínua, entre a mata contínua e a derrubada, entre o fragmento de 1 ha e as clareiras e entre a derrubada e as clareiras. $O$ dendrograma de dissimilaridade entre os ambientes é mostrado na figura 4. A mata contínua e as clareiras naturais formam um grupo à parte do grupo formado pelos fragmentos de 1 e 10 ha e a derrubada.

Houve uma correlação positiva entre os valores de dissimilaridade em espécies de vespas e os valores de dissimilaridade em espécies de abelhas entre os ambientes $(r=0,852 ;$ G.L. $=8 ; \mathrm{t}=4,603 ; \mathrm{p}<0,001)$.

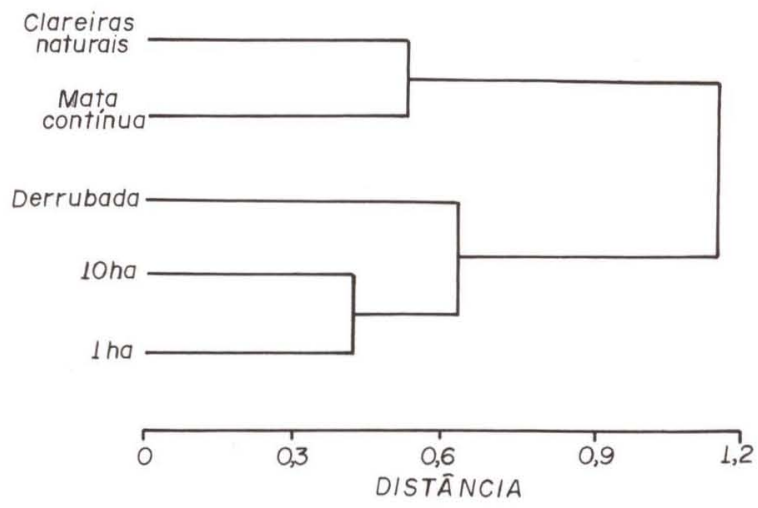

Fig. 4. Dendrograma de dissimilaridade entre os ambientes, em relação à fauna de abelhas.

\section{DISCUSSÃO}

O isolamento de uma porção de mata é seguido por modificações físicas que são responsáveis por muitas das modificações observadas nas comunidades de animais e plantas que aí existem, bem como na biologia de muitos desses organismos. Assim, a probabilidade de ocorrência e manutenção de diversas espécies pode 
ser afetada direta ou indiretamente pelas modificações microclimáticas produzidas pelo isolamento. Essas modificações se por um lado podem ser desfavoráveis a espécies adaptadas ao interior da mata, podem beneficiar espécies adaptadas a ambientes abertos, que podem então se estabelecerem no fragmento (LovEJOY et al. 1984, 1986).

Neste estudo, constatou-se que as vespas, de modo geral, fundaram ninhos mais freqüentemente nos locais de derrubada e nos fragmentos de mata menores. Esse resultado foi fortemente influenciado por duas espécies (Trypoxylon lactitarse e T. nitidum), as quais foram responsáveis pelo grande número de ninhos fundados nesses ambientes.

Houve, entretanto, espécies que fundaram seus ninhos preferencialmente na mata contínua, como é o caso das duas espécies de Podium.

Em um levantamento de Hymenoptera que nidificam em ninhos-armadilhas em um remanescente da Mata Atlântica, em Viçosa, Mina Gerais, R. Pérez-Maluf \& L.A. de O. Campos (informação pessoal) também constataram maior número de fundações de ninhos de vespas em locais abertos de vegetação secundária.

Sabe-se que entre as espécies de vespas que nidificam em cavidades preexistentes existem algumas que constroem seus ninhos preferencialmente em locais com pouca insolação (JAYASINGH \& TAFFE 1982). Há também espécies que fundam os seus ninhos em locais mais abertos. JENNINGS \& HoUSEWEART (1984) mostraram que vespas da família Eumenidae nidificam preferencialmente em habitats abertos, onde existe grande quantidade de plantas ruderais e flores em abundância.

Neste estudo, verificou-se que as abelhas apresentaram preferência pouco acentuada por fundarem mais ninhos na mata contínua e nas clareiras naturais. Este padrão contrastou com o observado para as vespas. Considerando que as clareiras são pequenas, a preferência exibida por determinadas espécies de abelhas por esses locais deve estar associada à existência de condições mais favoráveis ao desenvolvimento dos imaturos. O pólen e néctar utilizados no provisionamento dos ninhos têm de ser obtidos nas árvores da mata adjacente. Também entre as abelhas, houve espécies que fundaram mais ninhos na derrubada e nos fragmentos de mata pequenos e espécies que nidificaram sem uma preferência óbvia.

FYE (1972) relatou alterações no tamanho das populações de vespas e abelhas, incluindo espécies que nidificaram em ninhos-armadilhas, em uma floresta de clima temperado no Canadá, em razão das perturbações provocadas pelo desmatamento. Nos locais abertos e alterados as espécies de vespas e abelhas apresentaram populações maiores do que nos locais de mata não perturbada. $\mathrm{O}$ desmatamento criou áreas abertas, que foram colonizadas por um grande número de espécies de plantas pioneiras, as quais se constituíram em importante fonte de alimento para esses insetos, favorecendo o aumento de suas populações.

No presente estudo as espécies de abelhas adaptadas à mata contínua $(C$. terminata, Lagobata ornata, A. moratoi, Duckanthidium sp., M. orbiculata, Megachile (Rysochile) cfr. cara, Eufriesea purpurata e E. theresiae) mostraram ser mais sensíveis aos efeitos da fragmentação do seu ambiente que as espécies de vespas (Podium sexdentatum, P. rufipes, T. (Trypargilum) aff. nitidum, T. (Trypargilum) 
scrobiferum, T. (Trypargilum) sp. 2, T. (Trypoxylon) aff. ungicorne, Pisoxylon xanthosoma, Pison (Entomopison) sp., Pachodynerus sp., Zethus sp. e Auplopus sp.) (Fig. 2). Para o grupo de abelhas em questão, é possível que um fragmento de mata de 10 ha não ofereça condições suficientes para suportar populações com densidades semelhantes às existentes na mata contínua; no caso das espécies de vespas, um fragmento de 10 ha ainda é capaz de suportar tais populações. Contudo, nos fragmentos de 1 ha os efeitos da fragmentação foram também evidentes para essas espécies.

A maior sensibilidade das abelhas à fragmentação pode estar associada à diminuição de fontes de alimento, à falta de locais adequados para a nidificação nos ambientes mais alterados, ou às restrições impostas por seus limites de tolerância a fatores ambientais, como temperatura (PARKER 1986). Nos fragmentos de mata, a diversidade botânica deve ser menor que na mata contínua. Muitas plantas que são fontes importantes de alimento para essas espécies de abelhas podem estar ausentes, o que faz com que essas abelhas tenham de explorar fontes alternativas ou, ainda, competir com espécies que exploram de forma mais eficiente estas mesmas fontes. Isso pode ser um fator que provoca uma diminuição no tamanho de suas populações nos fragmentos de mata pequenos, embora não exclua essas espécies destes ambientes.

Como neste trabalho o número de espécies coletado foi pequeno e não muito variável entre os ambientes estudados, os valores de H' obtidos refletem, principalmente, mudanças nas proporções de cada espécie, ou seja, na dominância. A diversidade de espécies de vespas foi maior na mata contínua, nas clareiras naturais e nos fragmentos de 10 ha. Quanto às abelhas, a maior diversidade ocorreu na mata contínua. Os baixos valores dos índices de diversidade encontrados nos fragmentos de mata de 1 ha e nas áreas de derrubada refletem a elevada dominância na fundação de ninhos por parte de $T$. lactitarse e $T$. nitidum entre as vespas, e de $C$. dichrootricha, entre as abelhas.

A análise de similaridade revelou que tanto a fauna de vespas como a de abelhas da mata contínua são diferentes da fauna dos fragmentos e das áreas desmatadas. Houve, portanto, diferenças na composição de espécies da mata contínua e das áreas mais abertas e alteradas. Verificou-se que as clareiras naturais pareceram menos semelhantes às áreas desmatadas, em relação à composição faunística, do que à mata contínua. Isso mostrou que os grupos de vespas e abelhas que fundaram ninhos nos locais desmatados (Ampulex sp., P. (Pison) cressoni, $T$. (Trypargilum) fugax, T. (Trypargilum) aff. nitidum, Pachodynerus gianelli, E. gaianii e $C$. analis, principalmente) não foram os mesmos que fundaram ninhos nas clareiras naturais (P. xanthosoma, P. rufipes, P. sexdentatum, $T$. (Trypoxylon) aff. ungicorne, E. theresiae, A. moratoi e $M$. orbiculata, principalmente). A composição dos fragmentos foi alterada pela ocorrência de espécies de vespas e abelhas, que, aparentemente, são típicas de locais abertos e desmatados (por exemplo, T. (Trypargilum) lactitarse, $T$. (Trypargilum) nitidum e $C$. analis). Esse efeito foi mais pronunciado nos fragmentos de 1 ha. De um modo geral, locais que foram dissimilares com relação à fauna de vespas, também o foram com relação à fauna de abelhas. 
Com relação à fauna de vespas, os fragmentos de 1 ha foram mais semelhantes aos locais de derrubada do que à mata contínua. Os fragmentos de 10 ha foram mais semelhantes à mata contínua do que à derrubada. Com relação à fauna de abelhas, os fragmentos de 1 ha também foram mais semelhantes à derrubada do que a mata contínua. Os fragmentos de 10 ha foram tão semelhantes à mata contínua quanto à derrubada. Nos fragmentos de 1 ha, foi verificado grande número de fundações de ninhos por espécies, que também fundaram ninhos muito freqüentemente em locais desmatados. Possivelmente, fatores abióticos e bióticos dos fragmentos pequenos são semelhantes aos dos locais desmatados próximos, o que favorece a penetração nestes ambientes de espécies de áreas mais abertas.

Das espécies de abelhas estudadas, aquelas que parecem ser mais adaptadas à mata contínua foram mais sensíveis à fragmentação do que as espécies de vespas correspondentes, uma vez que fundaram um número proporcionalmente muito menor de ninhos nos fragmentos do que na mata contínua. Para as espécies de vespas, a redução no número de ninhos fundados entre a mata contínua e os fragmentos foi menos acentuada.

Áreas pequenas de mata são muito susceptíveis à imigração de animais e plantas de habitats secundários que as rodeiam, resultantes da ação antrópica (JANZEN 1983). Espécies da borda, que ocorrem com sucesso na interface entre habitats, preferem áreas pequenas que apresentam uma elevada relação perímetro/área. Por outro lado, as espécies intolerantes à borda serão mais bem sucedidas em reservas maiores, onde esta relação é menos acentuada. Espécies de borda ocorrerão com sucesso também em ambientes alterados com forte ação antrópica (DIAMOND \& MAY 1976).

MARTINS (1987) mostrou que para as espécies de Drosophila, que ocorrem em fragmentos de mata isolados do PDBFF, há ocorrência de espécies de mata contínua, de áreas abertas e espécies que, aparentemente, são indiferentes às características estruturais dos ambientes. MARTINS (1989) demonstrou também que a insularização da mata produziu modificações no interior dos fragmentos, suficientes para permitir a penetração de espécies de Drosophila, que são mais bem sucedidas em locais abertos e alterados. A composição faunística do interior da mata contínua era diferente da composição das áreas de derrubada e, nos fragmentos, foram coletadas, além de espécies de mata, espécies predominantes em áreas desmatadas. Fragmentos de 1 e 10 ha possuem alta freqüência de espécies que são dominantes nos locais abertos. O mesmo ocorreu no presente estudo.

O número de espécies de borboletas aumenta nos fragmentos de mata, em razão da invasão de espécies que são comuns na derrubada. Nos fragmentos de $1 \mathrm{e}$ 10 ha, as espécies típicas de mata tornaram-se raras com o passar do tempo e algumas até desapareceram. O aumento da intensidade luminosa nos fragmentos é favorável não apenas à atividade dessas espécies de borboletas de áreas abertas, mas também às plantas que são suas fontes de alimento (LovEJOY et al. 1984, 1986).

A penetração de espécies típicas de derrubada nos fragmentos reflete as alterações microclimáticas que ocorrem no seu interior após o isolamento. As condições nos fragmentos devem tornar-se mais semelhantes àquelas existentes nas áreas desmatadas. Fragmentos de 10 ha e menores devem ser bastante afetados por estas alterações (LOVEJOY et al. 1986). 
Como as espécies de abelhas adaptadas à mata parecem ser muito sensíveis ao processo de fragmentação, as comunidades de plantas floriferas dos fragmentos de mata menores podem, a longo prazo, sofrer modificações em sua composição, uma vez que um grande número de espécies vegetais depende das abelhas para a polinização de suas flores e reprodução. Contudo, é importante ressaltar que a interpretação dos efeitos da fragmentação deve ser feita com cautela, tendo em vista as variações naturais do ambiente que ocorrem no interior da própria mata contínua (SousA 1984).

As variações observadas no número de ninhos de vespas e abelhas coletados no interior da mata contínua foram, pelo menos em parte, conseqüência da pequena amostragem obtida; no entanto, podem também ser conseqüência dos padrões do mosaico ambiental existente dentro da própria mata. Mesmo quando as condições físicas de um ambiente são relativamente uniformes em uma região, são muito comuns variações espaciais nas taxas de reprodução, recrutamento, crescimento, e sobrevivência dos organismos (SoUSA 1984). Essas variações, por sua vez, podem ser conseqüência de variações na intensidade de interações biológicas, disponibilidade de recursos e condições microclimáticas.

Portanto, as variações existentes no interior da mata devem ser responsáveis, pelo menos por parte, das diferenças, de composição e abundância de animais, entre os locais.

AGRADECIMENTOS. Ao Prof. Pe. Jesus Santiago Moure (UFPR), Dr. Sérvio Túlio Amarante (MZUSP), Dr. Arnold S. Menke (Systematic Entomology Lab. - U.S. National Museum) e Dr. A. Willink (Facultad de Ciencias Naturales e Instituto Miguel Lillo - Universidad Nacional de Tucumán) pela identificação das espécies de abelhas e vespas. Este estudo foi parcialmente financiado pelo Instituto Nacional de Pesquisas da Amazônia (INPA) e pelo Smithsonian Tropical Research Institution (STRI) e representa a publicação número 308 da série técnica do projeto Dinâmica Biológica de Fragmentos Florestais (Instituto de Pesquisas da Amazônia, Caixa Postal 478, 69011-970 Manaus, Amazonas, Brasil).

\section{REFERÊNCIAS BIBLIOGRÁFICAS}

BATRA, S.W. 1984. Solitary bees. Sci. Amer. 250 (2): 86-93.

BierregAard JR., R.D. \& T. Lovejoy. 1988. Birds in Amazonian forest fragments: effects of insularization, p. 1564-1579. In: H. Quellet (Ed.). Acta XIX Cong. Int. Ornith.. Ottawa, Univ. of Ottawa Press, 1409p.

Cowan, D.P. 1991. The solitary and presocial Vespidae, p. 33-73. In: K.E. Ross \& R.W. Matthews (Ed.). The social biology of wasps. Ithaca, Comstock Publishing Associates, 678p.

DiAmond, J.M. \& R.M. M^Y. 1976. Island biogeography and the design of natural reserves, p. 163-186. In: R.M. MAY (Ed.). Theoretical ecology: principles and applications. Oxford, Blackwell, 317p.

Evans, H.E. 1966. The behavior patterns of solitary wasps. Ann. Rev. Entomol. 11: 123-54.

Evans, H.E. \& M.J.W. Eberhard. 1970. The Wasps. Ann. Arbor., The University of Michigan Press, $265 \mathrm{p}$.

FYE, R.E. 1972. The effect of forest disturbances on populations of wasps and bees in Northwestern Ontario (Hymenoptera: Aculeata). Canad. Entomol. 104: 1623-1633.

Henderson, A.; G. Gallano \& R. Bernal. 1995. Field guide to the palms of the Americas. New Jersey, Princeton University Press, 353p. 
Hutcheson, K. 1970. A test of comparing diversities based on the Shannon formula. Jour. Theor. Biol. 29: $151-154$.

JANZEN, D.H. 1983. No park is an island: increase in interference from outside as park size decreases.

Oikos 41: 402-410.

JAYASINGH, D.B. \& C.A. TAFFE. 1982. The biology of the eumenid mud-wasp Pachodynerus nasidens in trapnests. Ecological Entomology 7: 283-289.

JenNings, D.T. \& M.W. HousewarT. 1984. Predation by eumenid wasps (Hymenoptera: Eumenidae) on spruce budworm (Lepidoptera: Tortricidae) and other lepidopterous larvae in spruce-fir forests of Maine. Ann. Entomol. Soc. Amer. 77 (1): 39-45.

Krombein, K.V. 1960a. Biological notes on some Hymenoptera that nest in sumach pith. Ent. News 71 (2): 29-36.

-1960b. Biological notes on some Hymenoptera that nest in sumach pith. Ent. News 71 (3): 63-9.

1967. Trap-nesting wasps and bees: life histories, nests and associates. Washington, Smithsonian Press, 569p.

Lovejoy, T.E. 1980. Discontinuous wilderness: minimum areas for conservation. Parks 5 (2): 13-5.

LOVEJoy, T.E.; J.M. RANKIN; R.O. BierRegaARd JR.; K.S. Brown JR.; L.H. EMmONS \& M.E. VAN DER VOOR. 1984. Ecosystem decay of Amazon forest remnants, p. 295-325. In: M.H. NITECKI (Ed.). Extinctions. Chicago, University of Chicago, 354p.

Lovejoy, T.E.; R.O. BierRegaArd JR.; A.B. Rylands; J.R. Malcon; C.E. Quintela; L. Harper; K.S. Brown JR.; A.H. Powell; G.V.N. Powell; H.O.R. SCHUBART \& M.B. HAYS. 1986. Edge and other effects on isolation on Amazon forest fragments, p. 257-285. In: M.E. Soule (Ed.). Conservation biology: the science of scarcity and diversity. Sunderland, Massachusetts, Sinauer, 584p.

LUDWIG, J.A. \& J.F. REYNOLDS. 1988. Statistical ecology: a primer on methods and computing. New York, John Wiley \& Sons, 337p.

Magurran, A.E. 1988. Ecological diversity and its measurement. Princeton, Princeton University Press, $179 \mathrm{p}$.

MARTINS, M. 1987. Variação espacial e temporal de algumas espécies e grupos de Drosophila (Diptera) em duas reservas de mata isoladas nas vizinhanças de Manaus (Amazonas, Brasil). Bol. Mus. Par. Emílio Goeldi. Ser. Zool. 3 (2): 195-218.

- 1989. Invasão de fragmentos florestais por espécies oportunistas de Drosophila (Diptera, Drosophilidae). Acta Amazonica 19: 265-271.

PARKER, F.D. 1986. Factors influencing mortality and nesting in managed populations of the sunflower leafcutter bee (Hymenoptera: Megachilidae). Environ. Entomol. 15(4): 877-879.

PIELOU, E.C. 1984. The interpretation of ecological data: a primer on classification and ordination. New York, John Wiley \& Sons, 263p.

RouBIK, D.W. 1989. Ecology and natural history of tropical bees. Cambridge, Cambridge University Press, 514p.

SoKal, R.R. \& F.J. RoHLF. 1981. Biometry. San Francisco, Freeman and Company, 887p.

SousA, W.P. 1984. The role of disturbance in natural communities. Ann. Rev. Ecol. Syst. 15: 353-391.

Recebido em 06.VIII.1998; aceito em 25.IV.2000. 